University of Nebraska - Lincoln

DigitalCommons@University of Nebraska - Lincoln

$9-1-2006$

\title{
Single-crystal, polarized, Raman scattering study of the molecular and lattice vibrations for the energetic material cyclotrimethylene trinitramine
}

\author{
James C. Haycraft \\ University of Nebraska - Lincoln \\ Lewis L. Stevens \\ University of Nebraska - Lincoln \\ Craig J. Eckhardt \\ University of Nebraska - Lincoln, ceckhardt1@unl.edu
}

Follow this and additional works at: https://digitalcommons.unl.edu/chemistryeckhardt

Part of the Chemistry Commons

Haycraft, James C.; Stevens, Lewis L.; and Eckhardt, Craig J., "Single-crystal, polarized, Raman scattering study of the molecular and lattice vibrations for the energetic material cyclotrimethylene trinitramine" (2006). Craig J. Eckhardt Publications. 1.

https://digitalcommons.unl.edu/chemistryeckhardt/1

This Article is brought to you for free and open access by the Published Research - Department of Chemistry at DigitalCommons@University of Nebraska - Lincoln. It has been accepted for inclusion in Craig J. Eckhardt Publications by an authorized administrator of DigitalCommons@University of Nebraska - Lincoln. 


\title{
Single-crystal, polarized, Raman scattering study of the molecular and lattice vibrations for the energetic material cyclotrimethylene trinitramine
}

\author{
James J. Haycraft, Lewis L. Stevens, and Craig J. Eckhardt ${ }^{\mathrm{a})}$ \\ Department of Chemistry, University of Nebraska-Lincoln, Lincoln, Nebraska 68588-0304 \\ and Nebraska Center for Materials and Nanoscience, University of Nebraska-Lincoln, Lincoln, \\ Nebraska 68588-0304
}

(Received 27 January 2006; accepted 24 April 2006; published online 7 September 2006)

The single-crystal, polarized, Raman spectra for cyclotrimethylene trinitramine (RDX) have been studied at room temperature and pressure over the region of $5-3400 \mathrm{~cm}^{-1}$. While the observed Raman bands agree well with a previous Raman study, this work provides a consistent interpretation of the Raman spectra by assignment of fundamental modes, overtones, and combination bands. A total of 56 internal vibrations were observed, 36 of which were labeled as fundamental molecular vibrations. In addition, all 24 lattice optical phonons are identified. All six possible polarizations were probed to obtain the $A_{g}, B_{1 g}, B_{2 g}$, and $B_{3 g}$ symmetry modes for both the lattice and molecular modes. Finally, the importance of these phonons for coupling with "doorway" Raman-active modes of RDX is discussed in relation to proposed mechanisms for detonation initiation. (C) 2006 American Institute of Physics. [DOI: 10.1063/1.2234799]

\section{INTRODUCTION}

Although the phenomenology of detonation has been recognized for over a millennium, the understanding of detonation initiation from a microscopic, mechanistic viewpoint is limited. The complications that preclude a detailed determination of specific pathways for detonation initiation are rooted in the disparate time and length scales that distinguish the initial, shockwave-induced excitation from the subsequent decomposition in the detonation wave. Given that detonation ultimately results in molecular decomposition, the energy supplied by the shockwave must become available to the internal degrees of freedom of the molecule. The specific pathway by which the mechanical energy of the shockwave funnels into a molecular excitation, whether vibrational or electronic, is a current topic of debate. ${ }^{1-10}$

The goal of this research is to provide an unambiguous determination of the Raman-active vibrations for cyclotrimethylene trinitramine (RDX), a common secondary explosive. Secondary explosives are advantageous to study because they are relatively insensitive to mechanical initiation compared to primary explosives. The number of possible vibrational states available to shockwave activation in a secondary explosive normally exceeds that available in a primary explosive, which is often composed of small, ionic molecules.

For the shockwave-induced vibrational excitation of secondary explosives, a determination of lattice and molecular vibration anharmonicity is vital. A direct and quantitative assessment of mode anharmonicity is difficult to determine, but recent experimental ${ }^{11-13}$ reports have detailed the anharmonicity of specific vibrations in a few secondary explosives through the analysis of linewidths and their temperature de-

\footnotetext{
${ }^{a)}$ Electronic mail: eckhardt@unlserve.unl.edu
}

pendence. For secondary explosives, it has been proposed that low-energy molecular vibrations, termed "doorway modes," are critical to detonation initiation. ${ }^{5-7}$ These necessarily anharmonic vibrations provide the bridge between the shock-populated phonons of the material and the "uppumping" process through which the upper vibrational states of the molecule are increasingly populated to the point of decomposition. However, before a theory involving a complicated manifold of anharmonic vibrational interactions can be substantiated, the static vibrational properties of secondary explosives must first be experimentally well defined.

In order to provide an experimental foundation upon which vibrational models of detonation initiation can be built, a series of studies of the molecular and lattice vibrational properties need to be performed on several energetic materials. Recently, our laboratory published a comprehensive analysis on the molecular and lattice vibrational structure of the beta polymorph of the energetic material cyclotetramethylene tetranitramine ( $\beta$-HMX) at ambient conditions. ${ }^{14}$ The current report focuses on the external and internal vibrational properties of the room temperature polymorph of RDX. Additionally, comparisons can be made between HMX and RDX Raman spectra to examine if common vibrational properties exist and how these commonalities may relate to detonation initiation.

Much of the Raman work on RDX has dealt with field detectors that increase detection limits and provide ultrafast detection times. ${ }^{15}$ These topics are of increased importance given the current emphasis on baggage screening. None of these studies address the fundamental question of assignment of the modal symmetries of RDX. These symmetries are important in the analysis of the anharmonicity of the vibrational modes of RDX and for determining the details of the electronic-vibrational coupling that may be involved in detonation initiation. ${ }^{10}$ 
Two previous experimental polarized Raman studies report the vibrational structure of single crystals of RDX. ReyLafon et al. ${ }^{16}$ published the Raman spectrum of RDX from 200 to $3200 \mathrm{~cm}^{-1}$. They report a total of 39 vibrations for spectra at both room temperature and $453 \mathrm{~K}$ for RDX, its deuterated derivative, and ${ }^{15} \mathrm{~N}$ ring-substituted compound in an attempt to assign all the vibrational motions. In a separate publication, Rey-Lafon et al. ${ }^{17}$ examined the Raman spectrum of RDX from 10 to $200 \mathrm{~cm}^{-1}$. For the four different optical lattice mode symmetries, they report a total of 23 vibrations, with degeneracies claimed for the $51 \mathrm{~cm}^{-1} A_{g}$ mode and the $49 \mathrm{~cm}^{-1} B_{3 g}$ mode. In addition, they report several possible low-energy, molecular modes in the region from 86 to $159 \mathrm{~cm}^{-1}$.

In addition to these experimental reports, there also have been several calculations of the vibrational structure of RDX. Rice and Chabalowski ${ }^{18}$ performed an ab initio and a nonlocal density functional study on three possible conformers of the RDX molecule with various levels of computational refinement. They report the best overall fit with the experimental data of Rey-Lafon et al. ${ }^{16}$ using the B3LYP/6-311+G ${ }^{* *}$ basis set and the structural conformer that corresponds to the $C_{s}$ symmetry seen in the RDX solid. ${ }^{19}$ Luty et al. ${ }^{10}$ calculated bond lengths and angles for the RDX molecule using density functional theory (DFT) and found very good agreement with the study by Rice and Chabalowski. Additional calculations were performed to determine the normal vibrational frequencies of RDX, which are presented in Table IV in the supporting information of that publication. These vibrational frequencies were also in good agreement with those presented by Rice and Chabalowski. These calculational studies dealt solely with the molecular vibrations of RDX and provided no comparisons to the prior experimental study concerning the energies of the 23 observed optical phonons. This work reports all 24 lattice optical phonons and identifies fundamental molecular vibrations in assignments that are completely consistent with assigned combination bands and overtones in each of the four modal symmetries of the crystal.

In the next section, a brief discussion of the symmetry analysis of both the intra- and intermolecular modes is given. The subsequent section presents the experimental procedures and resulting spectra for all four symmetries represented by the six different possible polarizations. The following discussion analyzes these results in light of the previous studies so that assignments of the fundamental, overtone, and combination modes of the inter- and intramolecular vibrational transitions can be made entirely consistent. Finally, these results are discussed in relation to detonation initiation.

\section{SYMMETRY ANALYSIS}

The RDX free molecule possesses $C_{3 v}$ point group symmetry. ${ }^{20}$ The irreducible representations for the 57 vibrations of the free molecule are

$$
\Gamma_{\text {vib }}=12 A_{1}+7 A_{2}+19 E,
$$

where the $19 E$ modes are degenerate. However, it is known that RDX is distorted into nearly $C_{s}$ symmetry in the solid, ${ }^{19}$

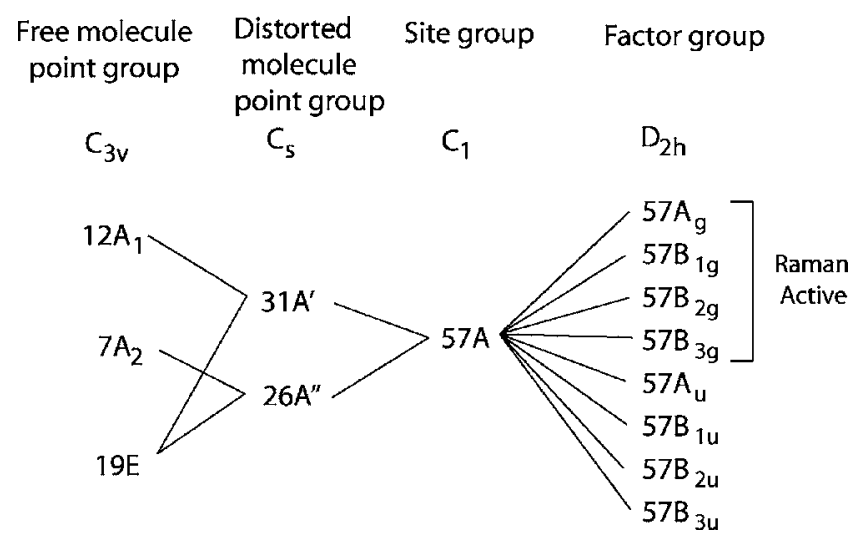

FIG. 1. Correlation diagram for the RDX internal modes.

so in order to correctly predict the number of vibrations seen in the Raman spectrum of crystalline RDX, this must be taken into account. Taking the $C_{3 v}$ symmetry of the free molecule and mapping it onto the pseudo- $C_{s}$ symmetry of RDX in the solid gives

$$
\Gamma_{\text {vib }}=31 A^{\prime}+26 A^{\prime \prime} \text {. }
$$

The RDX molecules are located at $C_{1}$ sites in the unit cell. Mapping the distorted $C_{s}$ symmetry of the molecule onto the $C_{1}$ site group of the unit cell gives

$$
\Gamma_{\text {vib }}=57 A \text {. }
$$

Finally, taking into account the factor group symmetry of $D_{2 h}$, we can map the $C_{1}$ site onto the unit cell to deduce the symmetries of the molecular vibrational modes of RDX. This expression is given as

$$
\begin{aligned}
\Gamma_{\text {vib }}= & 57 A_{g}+57 B_{1 g}+57 B_{2 g}+57 B_{3 g}+57 A_{u}+57 B_{1 u} \\
& +57 B_{2 u}+57 B_{3 u} .
\end{aligned}
$$

The gerade symmetry modes are Raman active. Thus, all symmetry information contained in the free molecule is lost because the eight RDX molecules in the unit cell sit at $C_{1}$ sites, which are general positions. This crystal-imposed symmetry reduction will lead to complicated spectra from which information about the parent vibrations of the free molecule will be nearly impossible to assign. This mapping procedure is summarized in the correlation diagram presented in Fig. 1.

Factor group analysis also gives the number and symmetries of the optical phonons. Applying this procedure to the $D_{2 h}$ unit cell with eight RDX molecules sitting at $C_{1}$ sites yields the following irreducible representations:

$$
\begin{aligned}
\Gamma_{\text {lattice }}= & 6 A_{g}+6 B_{1 g}+6 B_{2 g}+6 B_{3 g}+6 A_{u}+5 B_{1 u}+5 B_{2 u} \\
& +5 B_{3 u .}
\end{aligned}
$$

Only the 24 gerade modes are Raman active.

\section{EXPERIMENT}

Optical quality, untwinned, single crystals of RDX were grown evaporatively at $298 \mathrm{~K}$ from solutions of cyclohexanone saturated with de-ionized water. Crystals formed in the orthorhombic space group $P b c a$, with lattice constants $a$ $=13.182 \AA, b=11.574 \AA$, and $c=10.709 \AA$. The Pbca space 
group and lattice parameters were verified using singlecrystal x-ray diffraction and were equivalent, within error, to those reported by Choi and Prince. ${ }^{19}$ The crystals exhibited at least nine well-formed faces, eight of which were zonal to the $c$-crystallographic axis, $\{100\},\{010\},\{210\}$, and $\{-210\}$, with the remaining faces, $\{001\}$, orthogonal to the eight zonal faces. In order to assign the faces, the interfacial angles between all zonal and capping faces were determined by optical goniometry. The Miller indices of the faces, which were subsequently corroborated by single-crystal x-ray diffraction, are necessary to precisely define the scattering geometries used in the Raman scattering experiments.

Polarized Raman scattering experiments were performed on three single crystals of RDX at $295 \mathrm{~K}$ and ambient pressure to determine the frequencies and symmetries of the optical phonons and molecular modes. The polarizations of the incident and the scattered light were required to be along the axes of the optical indicatrix in order to determine the modal symmetries. Because RDX crystallizes in an orthorhombic space group, the axes of the optical indicatrix coincide with the crystallographic axes. The RDX crystals used in this study displayed $\{100\},\{010\}$, and $\{001\}$ faces, which allowed the axes of the optical indicatrix to be probed directly.

There are six unique polarization combinations for the incident and the scattered light, and the habit of the RDX crystals used in this study allowed all six polarizations to be directly probed. These six scattering geometries, written with a slight modification to the standard Porto notation ${ }^{21}$ where $x, y$, and $z$ have been replaced with the crystallographic axes, are $c(a a) b, c(b b) a, b(c c) a, b(a b) a, b(a c) a$, and $b(c b) a$, where the incident and scattered directions along the crystallographic axes are denoted by the letters before and after the parentheses, respectively, and the letters inside the parentheses denote the incident and scattered light polarizations. These scattering geometries allow for the determination of all possible modal symmetries.

Raman spectra were taken with $0.26 \mathrm{~W}$ of $532.0 \mathrm{~nm}$ light provided by a Coherent Verdi V-2 solid-state laser system. No degradation of the sample through localized heating was observed at this power setting. The dispersing element was a Spex Model 1401 monochromator operated in doublepass configuration equipped with dual 1800 grooves $/ \mathrm{mm}$ holographic gratings. The bandpass of the instrument ${ }^{22}$ at $532.0 \mathrm{~nm}$ was less than $1.0 \mathrm{~cm}^{-1}$. Calibration of the monochromator was achieved by using the known shift for the ring-breathing mode in liquid benzene ${ }^{23}$ located at $992.0 \mathrm{~cm}^{-1}$. All spectra were taken with a step size of $0.2 \mathrm{~cm}^{-1}$. The measured Raman shifts are accurate to within $\pm 1.0 \mathrm{~cm}^{-1}$.

\section{RESULTS}

The single-crystal, polarized, Raman spectra of RDX for the six scattering geometries were recorded over the range from 5 to $3400 \mathrm{~cm}^{-1}$. Given the large differences in Raman scattering intensities between molecular and lattice vibrations, the displayed spectra are separated into two regions: 200-3400 and 5-200 $\mathrm{cm}^{-1}$. The selection of these particular regions is not intended to indicate an absolute separation between the internal and external vibrational modes but rather as a means to easily view the vibrational structure in each displayed spectrum. However, for the region of $200-3400 \mathrm{~cm}^{-1}$, it will be shown in the next section that the observed bands arise solely from intramolecular vibrations. For the molecular vibrations, each of the four symmetries that arise from the correlation field splitting of eight RDX molecules in a $D_{2 h}$ lattice is shown in Fig. 2. The $A_{g}$ symmetry Raman internal vibrations for RDX are shown in parts (a)-(c). The remaining $B_{1 g}, B_{2 g}$, and $B_{3 g}$ symmetry molecular vibrations are shown, respectively, in Figs. 2(d)-2(f).

For the region from 5 to $200 \mathrm{~cm}^{-1}$, particular care is given to distinguish the low-energy molecular vibrations from the optical phonons. Similar to the displayed results for the molecular vibrations in the higher energy region, each of the six scattering geometries for the region of $5-200 \mathrm{~cm}^{-1}$ is shown in Fig. 3. The $A_{g}$ vibrations in this lower energy region are again shown in parts (a)-(c). The remaining $B_{1 g}$, $B_{2 g}$, and $B_{3 g}$ vibrations observed in this energy region are displayed, respectively, in Figs. 3(d)-3(f).

To aid in the assignment of the optical phonon energies and to separate the low-energy internal vibrations from the external vibrations, a decomposition of the spectral envelope from 15 to $200 \mathrm{~cm}^{-1}$ was performed. This peak decomposition of the six spectra shown in Fig. 3 was performed using a least squares fit with Lorentzian band shapes, and all decompositions matched the observed spectra with a minimum $r^{2}$ value of 0.99 . These results are shown in Fig. 4 . While a minimum number of bands were used to provide the best fit to the observed spectra, the addition of several bands that were not directly observed in the raw spectra was required to obtain an acceptable $r^{2}$ value. The symmetries and energy shifts determined in this work for the Raman-active, molecular vibrations of RDX over the range of $90-3400 \mathrm{~cm}^{-1}$ are listed in Table I, while the optical phonon energy shifts and associated symmetries are listed in Table II. In both tables, the values for the $A_{g}$ symmetry represent an average of the three different scattering geometries used to obtain the data for that modal symmetry.

\section{DISCUSSION}

\section{Molecular vibrations}

If crystalline RDX were composed of completely noninteracting molecules, the vibrational energies of the various lattice mode symmetries would be degenerate. However, inspection of Table I reveals that the average correlation field splitting that arises from intermolecular interactions is $1.1 \mathrm{~cm}^{-1}$. Frequency agreement with the previous experimental work is good, with an average deviation between the vibrational energies determined in this work and those previously reported being $1.5 \mathrm{~cm}^{-1}$. In addition, 16 vibrational modes are also identified.

The need to understand vibrational coupling emphasizes the importance of assigning possible combination and overtone bands in the Raman spectra of RDX. The previous Raman study of crystalline RDX only identified the energies of the Raman-active vibrations. However, it is important to 

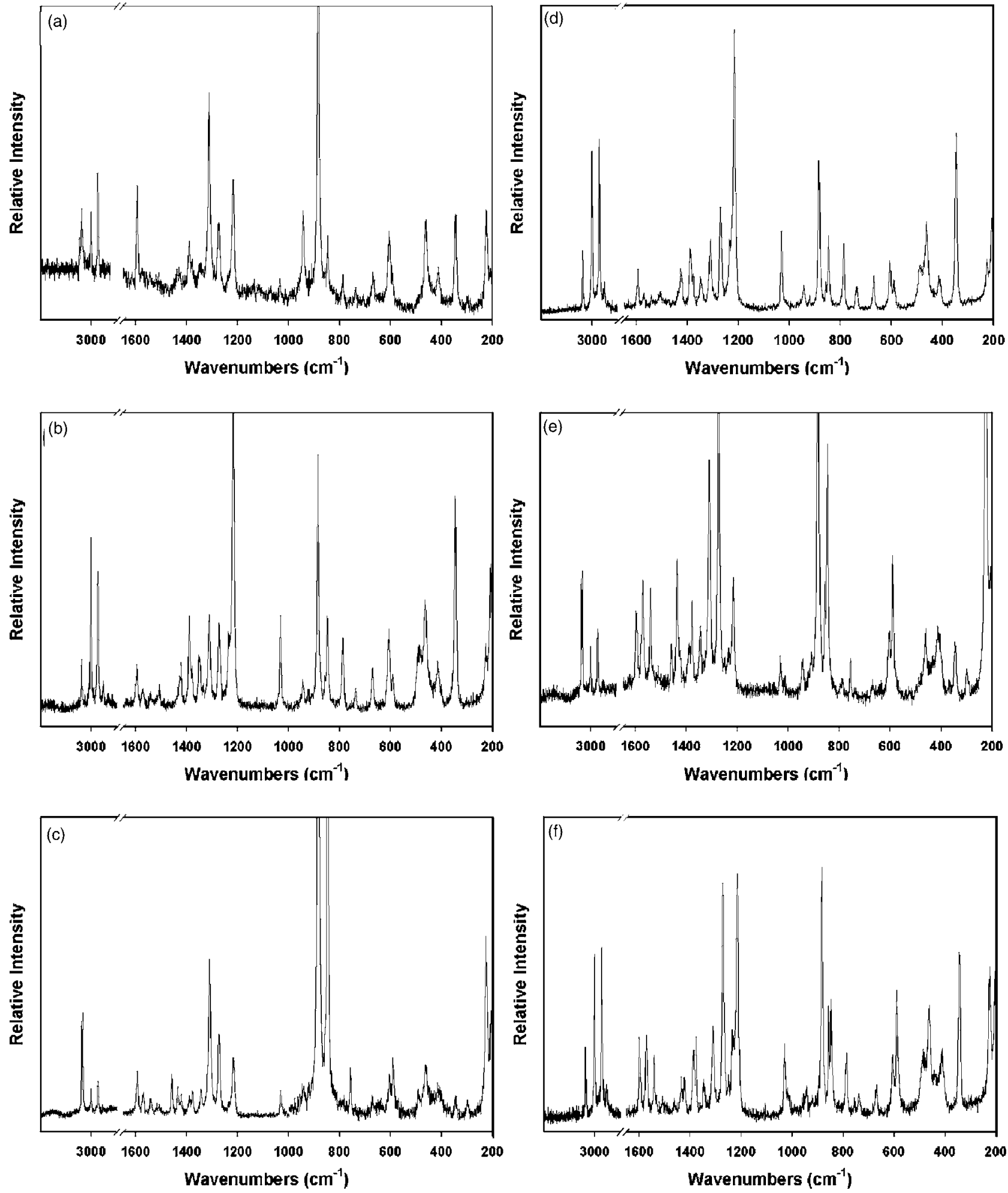

FIG. 2. Single-crystal, polarized, Raman spectra of RDX from 200 to $3400 \mathrm{~cm}^{-1}$. The modal symmetries are identified and the scattering geometries are given in modified Porto notation. (a) $A_{g}$ symmetry; scattering geometry: $c(a a) b$. (b) $A_{g}$ symmetry; scattering geometry: $c(b b) a$. (c) $A_{g}$ symmetry; scattering geometry: $b(c c) a$. (d) $B_{1 g}$ symmetry; scattering geometry: $b(a b) a$. (e) $B_{2 g}$ symmetry; scattering geometry: $b(a c) a$. (f) $B_{3 g}$ symmetry; scattering geometry: $b(c b) a$.

assign the fundamental transitions as well as the combination and overtone bands. For any analysis and comparison to calculations, it is crucial to assign the fundamental modes of vibration. This was achieved by assigning as fundamentals, the bands displaying the greater intensities and bands that could not be obtained as multiples, sums, or differences of frequencies of other observed bands. An additional criterion was that the difference in energy between the assigned and observed combination or overtone bands was kept to approximately $5 \mathrm{~cm}^{-1}$ or less, with the energy of the "calculated" combination or overtone band usually being higher than that of the observed. This is consistent with the anharmonic contraction of vibrational energy-level spacings with increasing vibrational quantum numbers. 

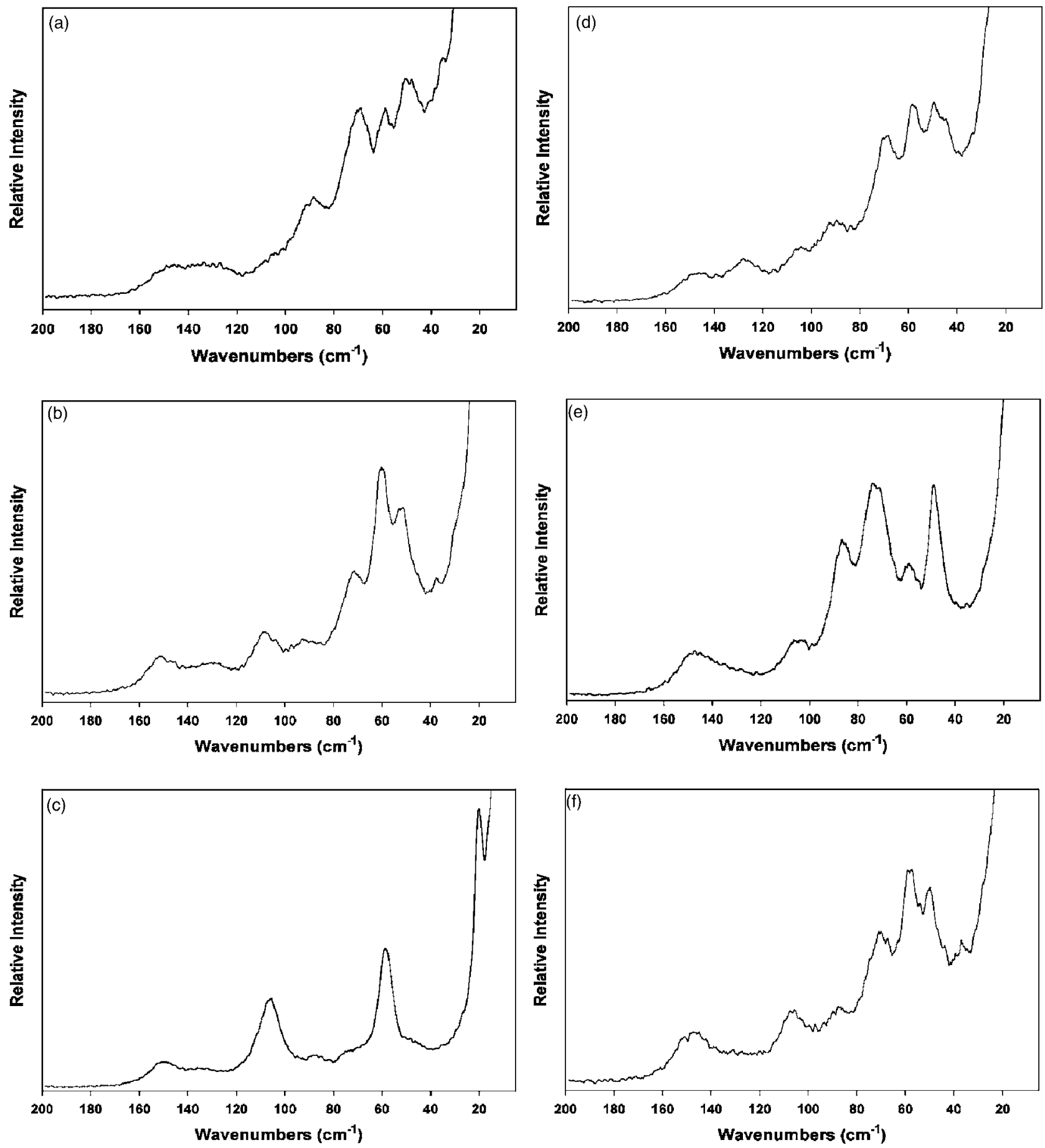

FIG. 3. Single-crystal, polarized, Raman spectra of RDX from 5 to $200 \mathrm{~cm}^{-1}$. The modal symmetries are identified and the scattering geometries are given in modified Porto notation. (a) $A_{g}$ symmetry; scattering geometry: $c(a a) b$. (b) $A_{g}$ symmetry; scattering geometry: $c(b b) a$. (c) $A_{g}$ symmetry; scattering geometry: $b(c c) a$. (d) $B_{1 g}$ symmetry; scattering geometry: $b(a b) a$. (e) $B_{2 g}$ symmetry; scattering geometry: $b(a c) a$. (f) $B_{3 g}$ symmetry; scattering geometry: $b(c b) a$.

The $C_{1}$ site group symmetry and eight molecules in the unit cell obviate any possibility of assigning the molecular modes from which the crystal modes are derived from. The crystal modal symmetries represent the Davydov-split components of the originating molecular transition. Thus, for a given mode, assignments are made to be consistent with the observed symmetry. For example, the $299.6 \mathrm{~cm}^{-1} A_{g}$ mode is assigned as an overtone while the related Davydov-split components are listed as combination bands. It is likely that all the $\sim 300 \mathrm{~cm}^{-1}$ modes arise from a molecular overtone, but without the Raman vibrational spectrum of the free molecule, which would clarify the nature of the originating molecular transitions, assignment is made to retain consistency with the observed modal symmetry.

Following our criteria of analysis, $36 A_{g}, 34 B_{1 g}, 36 B_{2 g}$, and $36 B_{3 g}$ symmetry fundamentals were identified. These fundamental transitions and the assignments for the combination and overtone bands are listed in Table I. A majority of the previously unreported modes observed in this work are assigned as combination bands. Given the inherently weak 

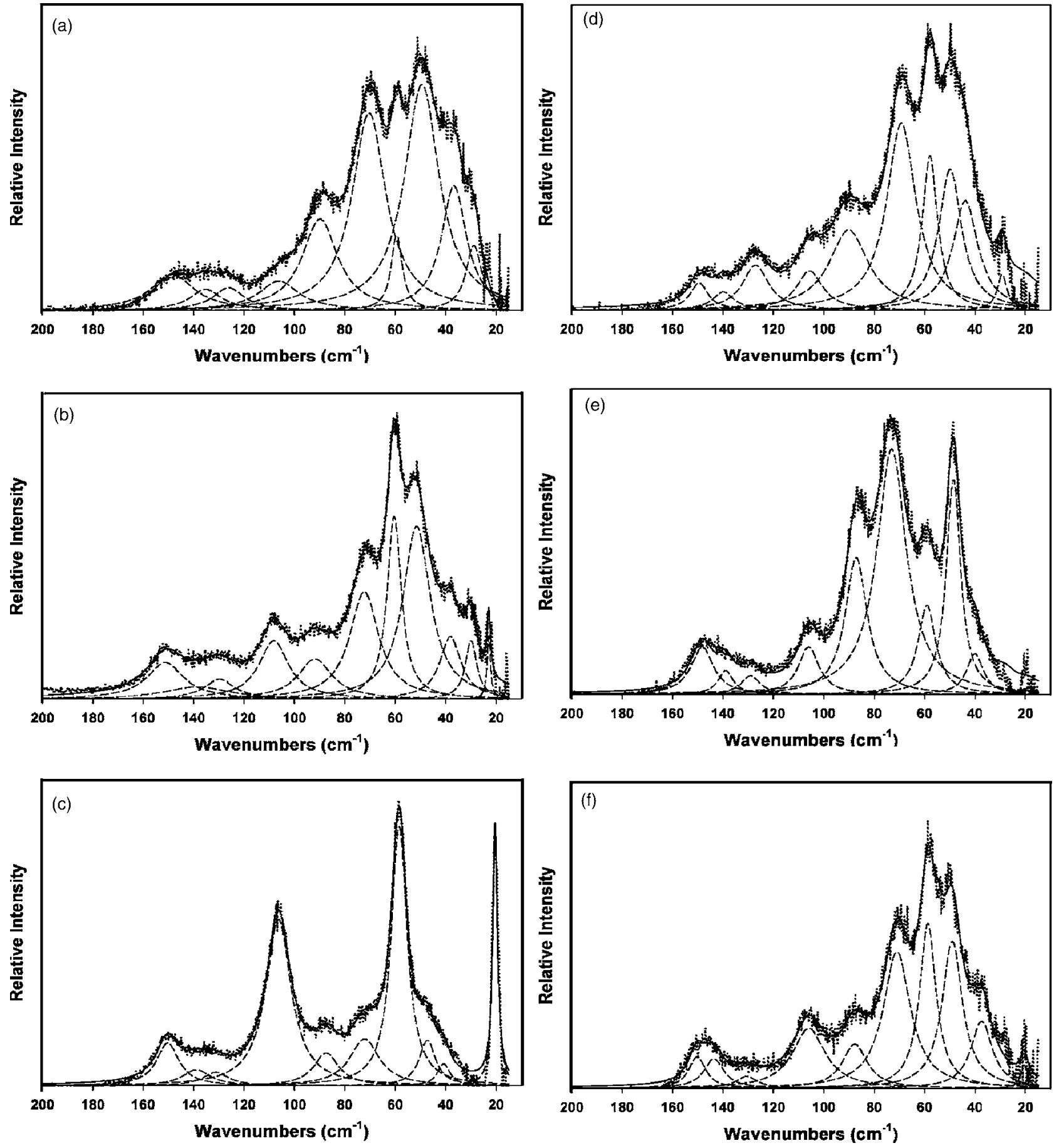

FIG. 4. Decomposition of the single-crystal, polarized, Raman spectra of RDX from 15 to $200 \mathrm{~cm}^{-1}$. A baseline has been subtracted from each spectrum to aid in peak identification. The dotted line represents the experimental data, also shown in Fig. 3. The dashed lines represent the resolved peaks under the band envelope. The black line is the sum of the constituent peaks' envelopes. (a) $A_{g}$ symmetry; scattering geometry: $c(a a) b$. (b) $A_{g}$ symmetry; scattering geometry: $c(b b) a$. (c) $A_{g}$ symmetry; scattering geometry: $b(c c) a$. (d) $B_{1 g}$ symmetry; scattering geometry: $b(a b) a$. (e) $B_{2 g}$ symmetry; scattering geometry: $b(a c) a$. (f) $B_{3 g}$ symmetry, scattering geometry: $b(c b) a$.

scattering intensity of these modes, it is not surprising they were overlooked in earlier work. Nevertheless, such modes are invaluable in generating a consistent set of mode assignments.

The rather intense molecular vibrations of RDX between 90 and $150 \mathrm{~cm}^{-1}$ are of particular interest. These are in an energy region that suggests they may serve as doorway modes according to the model of vibrational up-pumping detailed by Dlott and Fayer. A doorway mode is the proposed bridge by which the energy of the initial shockwave can pass from the external degrees of freedom of the energetic material into those of the molecule. Vibrations in this range were observed in the Raman spectra previously reported by ReyLafon et al.; however, they were only reported at an elevated temperature of $453 \mathrm{~K}$. These low-energy vibrations were clearly observed for RDX under ambient conditions in this work. Furthermore, following our assignment criteria, no viable candidates arose for assigning these low-energy vibra- 
TABLE I. RDX Raman-active molecular vibrational modes observed in this work and in that of Rey-Lafon et al. Modal symmetries, fundamental modes, and combination bands are given. Units are in $\mathrm{cm}^{-1}$, with the following abbreviations used to denote relative peak intensities: very strong (vs), strong (s), medium $(\mathrm{m})$, weak (w), very weak (vw). The absence of a mode in the particular polarization is denoted by a dash (-).

\begin{tabular}{|c|c|c|c|c|c|c|c|c|}
\hline \multicolumn{8}{|c|}{ This work } & \multirow{2}{*}{$\begin{array}{c}\text { Rey-Lafon } \\
\text { et al. }{ }^{\mathrm{a}}\end{array}$} \\
\hline \multicolumn{2}{|c|}{$A_{g}$} & \multicolumn{2}{|r|}{$B_{1 g}$} & \multicolumn{2}{|r|}{$B_{2 g}$} & \multicolumn{2}{|r|}{$B_{3 g}$} & \\
\hline $3077.3(\mathrm{~m})$ & Fundamental & $3075.6(\mathrm{~m})$ & Fundamental & 3077.8 (m) & Fundamental & 3076.8 (m) & Fundamental & 3075 \\
\hline $3068.5(\mathrm{~m})$ & Fundamental & $3067.6(w)$ & Fundamental & $3067.8(\mathrm{~m})$ & Fundamental & $3066.8(\mathrm{~m})$ & Fundamental & 3067 \\
\hline $3002.7(\mathrm{~m})$ & Fundamental & $3002.0(\mathrm{~s})$ & Fundamental & $3001.8(\mathrm{~m})$ & Fundamental & $3001.8(\mathrm{~s})$ & Fundamental & 3001 \\
\hline $2949.5(\mathrm{~m})$ & Fundamental & $2947.6(\mathrm{~s})$ & Fundamental & $2948.6(\mathrm{~m})$ & Fundamental & $2948.2(\mathrm{~s})$ & Fundamental & 2949 \\
\hline $2907.6(w)$ & $1311 A_{g}+1594 A_{g}$ & $2907.0(w)$ & $1311 A_{g}+1595 B_{1 g}$ & $2907.2(\mathrm{vw})$ & $1311 A_{g}+1594 B_{2 g}$ & $2906.4(w)$ & $1311 A_{g}+1594 B_{3 g}$ & - \\
\hline $1596.3(\mathrm{~m})$ & Fundamental & - & & $1599.2(\mathrm{~m})$ & Fundamental & $1600.0(\mathrm{~s})$ & Fundamental & - \\
\hline $1594.0(\mathrm{~m})$ & Fundamental & $1594.6(\mathrm{~m})$ & Fundamental & $1594.4(\mathrm{~m})$ & Fundamental & $1594.0(\mathrm{~m})$ & Fundamental & 1593 \\
\hline $1569.0(\mathrm{~m})$ & Fundamental & $1569.8(\mathrm{~m})$ & Fundamental & $1571.4(\mathrm{~s})$ & Fundamental & $1571.6(\mathrm{~s})$ & Fundamental & 1570 \\
\hline $1541.1(\mathrm{~m})$ & Fundamental & $1541.4(w)$ & Fundamental & $1540.2(\mathrm{~s})$ & Fundamental & $1540.8(\mathrm{~m})$ & Fundamental & 1538 \\
\hline $1515.8(\mathrm{vw})$ & $1311 A_{g}+208 A_{g}$ & - & & - & & - & & - \\
\hline $1507.9(\mathrm{w})$ & $1596 A_{g}-90 A_{g}$ & $1506.8(w)$ & $1595 B_{1 g}-90 A_{g}$ & $1509.8(\mathrm{vw})$ & $1594 B_{2 g}-90 A_{g}$ & $1507.2(w)$ & $1594 B_{3 g}-90 A_{g}$ & 1508 \\
\hline $1458.4(\mathrm{~m})$ & Fundamental & - & & $1459.4(\mathrm{~m})$ & Fundamental & $1461.4(w)$ & Fundamental & 1456 \\
\hline $1435.9(\mathrm{~m})$ & Fundamental & $1435.6(w)$ & Fundamental & $1435.8(\mathrm{~s})$ & Fundamental & $1435.0(\mathrm{~m})$ & Fundamental & 1433 \\
\hline $1427.6(\mathrm{vw})$ & $943 A_{g}+484 A_{g}$ & $1425.2(\mathrm{~m})$ & $943 A_{g}+483 B_{1 g}$ & - & & - & & - \\
\hline $1422.3(\mathrm{w})$ & $1273 A_{g}^{g}+150 A_{g}^{g}$ & - & & $1421.8(w)$ & $1273 A_{g}+148 B_{2 g}$ & $1422.2(\mathrm{~m})$ & $1273 A_{g}+150 B_{3 g}$ & 1422 \\
\hline $1388.8(\mathrm{~m})$ & Fundamental & $1390.0(\mathrm{~s})$ & Fundamental & $1389.8(\mathrm{~m})$ & Fundamental & $1386.4(\mathrm{~s})$ & Fundamental & 1387 \\
\hline $1376.8(\mathrm{~m})$ & Fundamental & $1376.2(\mathrm{~m})$ & Fundamental & $1377.2(\mathrm{~m})$ & Fundamental & $1377.0(\mathrm{~s})$ & Fundamental & 1377 \\
\hline $1353.2(w)$ & $1217 A_{g}+139 A_{g}$ & $1349.2(\mathrm{~m})$ & $1216 B_{1 g}+139 A_{g}$ & - & & $1348.6(\mathrm{~m})$ & $1215 B_{3 g}+139 A_{g}$ & - \\
\hline $1344.2(w)$ & $1217 A_{g}^{\circ}+129 A_{g}^{\circ}$ & - & & $1344.2(\mathrm{~m})$ & $1217 A_{g}+129 B_{2 g}$ & - & & 1346 \\
\hline $1334.0(\mathrm{vw})$ & $1541 A_{g}^{g}-208 A_{g}^{8}$ & $1337.0(w)$ & $1541 B_{1 g}-208 A_{g}$ & $1334.0(w)$ & $1540 B_{2 g}-208 A_{g}$ & - & & - \\
\hline $1311.4(\mathrm{~s})$ & Fundamental & $1309.4(\mathrm{~s})$ & Fundamental & 1311.0 (vs) & Fundamental & $1310.2(\mathrm{~s})$ & Fundamental & 1309 \\
\hline $1273.2(\mathrm{~s})$ & Fundamental & $1270.6(\mathrm{~s})$ & Fundamental & 1273.8 (vs) & Fundamental & 1271.3 (vs) & Fundamental & 1273 \\
\hline $1236.7(\mathrm{vw})$ & $1031 A_{g}+208 A_{g}$ & $1236.3(\mathrm{vw})$ & $1031 B_{1 g}+208 A_{g}$ & $1236.9(\mathrm{vw})$ & $1031 B_{2 g}+208 A_{g}$ & $1235.2(\mathrm{~m})$ & $1030 B_{3 g}+208 A_{g}$ & - \\
\hline $1230.2^{\mathrm{b}}(\mathrm{w})$ & $885 A_{g}^{\circ}+345 A_{g}^{\circ}$ & $1230.0^{\mathrm{b}}(\mathrm{m})$ & $885 A_{g}+346 B_{1 g}$ & $1229.4^{\mathrm{b}}(\mathrm{vw})$ & $885 A_{g}+346 B_{2 g}$ & $1230.0^{\mathrm{b}}(\mathrm{m})$ & $885 A_{g}+345 B_{3 g}$ & 1232 \\
\hline $1222.5^{\mathrm{b}}(\mathrm{vw})$ & $1569 A_{g}-345 A_{g}$ & $1225.0^{\mathrm{b}}(\mathrm{vw})$ & $1569 A_{g}^{\circ}-346 B_{1 g}$ & $1221.9^{\mathrm{b}}(\mathrm{w})$ & $1569 A_{g}-346 B_{2 g}$ & $1222.0^{\mathrm{b}}(\mathrm{m})$ & $1569 A_{g}-345 B_{3 g}$ & - \\
\hline $1217.0(\mathrm{~s})$ & Fundamental & 1216.4 (vs) & Fundamental & $1215.9(\mathrm{~s})$ & Fundamental & 1215.1 (vs) & Fundamental & 1214 \\
\hline $1031.7(\mathrm{~m})$ & Fundamental & $1030.8(\mathrm{~s})$ & Fundamental & $1030.6(\mathrm{~m})$ & Fundamental & $1029.6(\mathrm{~s})$ & Fundamental & 1029 \\
\hline $1017.6(\mathrm{vw})$ & $671 A_{g}+345 A_{g}$ & $1017.3(\mathrm{vw})$ & $671 A_{g}+346 B_{1 g}$ & $1018.0(\mathrm{vw})$ & $671 A_{g}+346 B_{2 g}$ & $1017.2(\mathrm{w})$ & $671 A_{g}+345 B_{3 g}$ & - \\
\hline $942.9(\mathrm{~m})$ & Fundamental & $944.6(\mathrm{~m})$ & Fundamental & $942.6(\mathrm{~m})$ & Fundamental & $942.8(\mathrm{~m})$ & Fundamental & 943 \\
\hline $920.7(w)$ & $1388 A_{g}-464 A_{g}$ & $920.6(w)$ & $1388 A_{g}-463 B_{1 g}$ & $920.6(w)$ & $1388 A_{g}-462 B_{2 g}$ & $921.2(\mathrm{vw})$ & $1388 A_{g}-463 B_{3 g}$ & 920 \\
\hline 908.4 (vw) & $493 A_{g}+415 A_{g}$ & - & & $909.2(\mathrm{w})$ & $493 A_{g}^{s}+414 B_{2 g}$ & $907.2(\mathrm{vw})$ & $493 A_{g}+414 B_{3 g}$ & - \\
\hline 885.0 (vs) & Fundamental & 884.4 (vs) & Fundamental & 883.2 (vs) & Fundamental & $883.8(\mathrm{vs})$ & Fundamental & 884 \\
\hline $857.7(\mathrm{~s})$ & Fundamental & $857.2(\mathrm{~m})$ & Fundamental & $857.4(\mathrm{~m})$ & Fundamental & $857.4(\mathrm{~s})$ & Fundamental & 855 \\
\hline 847.5 (vs) & Fundamental & $846.6(\mathrm{~s})$ & Fundamental & $846.6(\mathrm{~s})$ & Fundamental & $847.4(\mathrm{~s})$ & Fundamental & 847 \\
\hline $787.4(\mathrm{~m})$ & Fundamental & $787.6(\mathrm{~s})$ & Fundamental & $786.2(w)$ & Fundamental & $786.6(\mathrm{~m})$ & Fundamental & 786 \\
\hline $778.4(w)$ & $885 A_{g}-107 A_{g}$ & - & & - & & $778.6(w)$ & $885 A_{g}-106 B_{3 g}$ & - \\
\hline $755.8(w)$ & $606 A_{g}^{g}+150 A_{g}^{g}$ & - & & $755.4(w)$ & $606 A_{g}+148 B_{2 g}$ & $756.6(w)$ & $606 A_{g}+150 B_{3 g}$ & 756 \\
\hline $737.6(\mathrm{vw})$ & $606 A_{g}^{g}+129 A_{g}^{8}$ & $736.2(\mathrm{~m})$ & $129 A_{g}+606 B_{1 g}$ & $735.4(\mathrm{vw})$ & $129 A_{g}^{8}+605 B_{2 g}$ & $738.0(\mathrm{w})$ & $129 A_{g}^{8}+605 B_{3 g}$ & 739 \\
\hline $670.7(w)$ & Fundamental & $668.4(\mathrm{~m})$ & Fundamental & $669.6(\mathrm{w})$ & Fundamental & $667.4(\mathrm{w})$ & Fundamental & 669 \\
\hline $638.2(\mathrm{vw})$ & $415 A_{g}+225 A_{g}$ & - & & $635.2(\mathrm{vw})$ & $415 A_{g}+225 B_{2 g}$ & - & & - \\
\hline $606.2(\mathrm{~m})$ & Fundamental & $606.0(\mathrm{~s})$ & Fundamental & $604.8(\mathrm{~m})$ & Fundamental & $605.2(\mathrm{~m})$ & Fundamental & 605 \\
\hline $591.3(\mathrm{~m})$ & Fundamental & $590.0(\mathrm{~m})$ & Fundamental & $590.4(\mathrm{~s})$ & Fundamental & $590.2(\mathrm{~s})$ & Fundamental & 589 \\
\hline $493.0^{\mathrm{b}}(\mathrm{w})$ & Fundamental & $492.0^{\mathrm{b}}(\mathrm{s})$ & Fundamental & $492.0^{\mathrm{b}}(\mathrm{w})$ & Fundamental & $493.0^{\mathrm{b}}(\mathrm{m})$ & Fundamental & - \\
\hline $484.0^{\mathrm{b}}(\mathrm{m})$ & Fundamental & $483.2^{\mathrm{b}}(\mathrm{s})$ & Fundamental & $483.0^{\mathrm{b}}(\mathrm{w})$ & Fundamental & $483.0^{\mathrm{b}}(\mathrm{m})$ & Fundamental & 486 \\
\hline $464.0(\mathrm{~m})$ & Fundamental & $463.2(\mathrm{~s})$ & Fundamental & $461.8(\mathrm{~m})$ & Fundamental & $463.0(\mathrm{~s})$ & Fundamental & 463 \\
\hline $442.0^{\mathrm{b}}(\mathrm{vw})$ & $591 A_{g}-150 A_{g}$ & $441.0^{\mathrm{b}}(\mathrm{vw})$ & $591 A_{g}-150 B_{1 g}$ & $444.0^{\mathrm{b}}(\mathrm{vw})$ & $591 A_{g}-148 B_{2 g}$ & $439.0^{\mathrm{b}}(\mathrm{m})$ & $591 A_{g}-150 B_{3 g}$ & 440 \\
\hline $414.8(\mathrm{~m})$ & Fundamental & $414.8(\mathrm{~m})$ & Fundamental & $414.4(\mathrm{~m})$ & Fundamental & $414.0(\mathrm{~m})$ & Fundamental & 414 \\
\hline $344.8(\mathrm{~m})$ & Fundamental & 345.8 (vs) & Fundamental & $346.0(\mathrm{~m})$ & Fundamental & 344.6 (vs) & Fundamental & 347 \\
\hline $299.6(w)$ & $2\left(150 A_{g}\right)$ & $298.6(\mathrm{vw})$ & $150 A_{g}+150 B_{1 g}$ & $300.4(w)$ & $150 A_{g}+148 B_{2 g}$ & $298.4(\mathrm{vw})$ & $150 A_{g}+150 B_{3 g}$ & 301 \\
\hline $225.4(\mathrm{~s})$ & Fundamental & $226.4(\mathrm{~s})$ & Fundamental & 225.0 (vs) & Fundamental & $225.2(\mathrm{vs})$ & Fundamental & 224 \\
\hline $207.5(\mathrm{~s})$ & Fundamental & $206.8(\mathrm{~s})$ & Fundamental & $206.6(\mathrm{~s})$ & Fundamental & 204.8 (vs) & Fundamental & 205 \\
\hline $150.3(\mathrm{~s})$ & Fundamental & $149.5(\mathrm{~s})$ & Fundamental & $148.2(\mathrm{~s})$ & Fundamental & $150.2(\mathrm{~s})$ & Fundamental & $159^{\mathrm{c}}$ \\
\hline $139.0(\mathrm{~s})$ & Fundamental & 139.9 (s) & Fundamental & 138.9 (s) & Fundamental & $143.6(\mathrm{~s})$ & Fundamental & $144^{\mathrm{c}}$ \\
\hline $129.5(\mathrm{~s})$ & Fundamental & $127.0(\mathrm{~s})$ & Fundamental & $129.0(\mathrm{~s})$ & Fundamental & $130.6(\mathrm{~s})$ & Fundamental & $133^{\mathrm{c}}$ \\
\hline 107.3 (vs) & Fundamental & 105.6 (vs) & Fundamental & $106.0(\mathrm{~s})$ & Fundamental & 105.9 (vs) & Fundamental & 106 \\
\hline $90.0(\mathrm{~s})$ & Fundamental & 90.1 (vs) & Fundamental & 87.1 (vs) & Fundamental & 87.7 (vs) & Fundamental & 89 \\
\hline
\end{tabular}

${ }^{\mathrm{a}}$ Data taken from Refs. 16 and 17.

${ }^{\mathrm{b}}$ Modes positively identified through peak decomposition.

${ }^{c}$ Modes observed by Rey-Lafon et al. only at $453 \mathrm{~K}$. 
tions of RDX to lattice combination or overtone modes. With these considerations, assignment of the Raman-active fundamental internal modes of RDX has been achieved.

\section{Lattice modes}

While a detailed assessment of the molecular vibrations is required for an understanding of the decomposition pathways of the molecule, a thorough description of the optical phonons is equally important for insight into how the energy of the shockwave is initially transferred to the material. Additionally, a complete description of the lattice vibrations of RDX must also include an analysis of the acoustic phonons. These were obtained by Brillouin scattering using an apparatus and techniques reported elsewhere. ${ }^{24}$

From the symmetry analysis for the $D_{2 h}$ lattice of RDX, six optical phonons are expected to be found in four symmetries, $A_{g}, B_{1 g}, B_{2 g}$, and $B_{3 g}$. While six $A_{g}$ symmetry optical phonons are reported by Rey-Lafon et al., two of them have identical energies. Similarly, two "degenerate" optical phonons are listed with $B_{3 g}$ symmetry. The reason for making these degenerate assignments is unclear, and our measurements and data provide no support for them.

Inspection of Table II reveals that several optical phonons of different symmetries have similar energies within the error of their assignment $\left( \pm 1 \mathrm{~cm}^{-1}\right)$. These accidental degeneracies between different symmetry lattice vibrations are not in agreement with the observations of Rey-Lafon et $a l$. where they reported degeneracies within the $A_{g}$ and $B_{3 g}$ symmetry vibrations. These accidental degeneracies cannot be attributed to the correlation field splitting that gives rise to the comparable energies found for the molecular vibrations of disparate symmetries. Such accidental degeneracies are not without precedent. In two previous Raman scattering studies of $\beta$-HMX, an energetic material similar to RDX, an occurrence of accidentally degenerate optical phonons was reported. ${ }^{14,25}$

To be assured that these are accidental degeneracies of RDX, the possibility of breakthrough scattering must be considered. Breakthrough scattering has been used as an explanation of the appearance of disparate symmetries in a given vibrational spectrum. The observation of breakthrough bands is attributed to (1) a slight misalignment of the incident or scattering polarization or (2) a defect-induced change of the incident or scattering polarization. Each of these two cases was addressed in this Raman scattering study. For the first possibility, the polarizations of the incident and the scattered light were deliberately misaligned in an attempt to induce breakthrough scattering. In all cases, the observed Raman spectra displayed only minor differences from the spectra taken with correctly aligned incident and scattering polarizations. For the second possibility, defect-induced changes in a spectrum are necessarily a function of the quality of the crystal used. For our Raman studies three single crystals of RDX were used. The spectra obtained from each crystal were indistinguishable. Given these considerations, it is unlikely that the observed optical phonons are actually manifestations of breakthrough scattering. Instead, these lattice vibrations, like those observed for $\beta$-HMX, are accidentally degenerate. If
TABLE II. RDX Raman-active lattice optical modes observed in this work and in that of Rey-Lafon et al. Units are in $\mathrm{cm}^{-1}$. The absence of a mode in the particular polarization is denoted by a dash (-).

\begin{tabular}{|c|c|c|c|}
\hline \multicolumn{2}{|c|}{ This work } & \multicolumn{2}{|c|}{ Rey-Lafon et al. ${ }^{\text {a }}$} \\
\hline \multicolumn{4}{|c|}{$A_{g}$} \\
\hline 21.8 & Lattice & 20 & Lattice \\
\hline 29.4 & Lattice & 33 & Lattice \\
\hline 38.8 & Lattice & $51^{\mathrm{b}}$ & Lattice \\
\hline 49.6 & Lattice & $51^{\mathrm{b}}$ & Lattice \\
\hline 59.5 & Lattice & 59 & Lattice \\
\hline 71.9 & Lattice & 70 & Lattice \\
\hline \multicolumn{4}{|c|}{$B_{1 g}$} \\
\hline 20.2 & Lattice & 20 & Lattice \\
\hline 29.2 & Lattice & 28 & Lattice \\
\hline 43.8 & Lattice & 46 & Lattice \\
\hline 49.9 & Lattice & 52 & Lattice \\
\hline 57.9 & Lattice & 59 & Lattice \\
\hline 69.2 & Lattice & 70 & Lattice \\
\hline \multicolumn{4}{|c|}{$B_{2 g}$} \\
\hline 18.9 & Lattice & 20 & Lattice \\
\hline 28.0 & Lattice & 29 & Lattice \\
\hline 40.0 & Lattice & - & Lattice \\
\hline 48.5 & Lattice & 49 & Lattice \\
\hline 59.1 & Lattice & 60 & Lattice \\
\hline 73.0 & Lattice & 74 & Lattice \\
\hline \multicolumn{4}{|c|}{$B_{3 g}$} \\
\hline 20.5 & Lattice & 19 & Lattice \\
\hline 37.4 & Lattice & 37 & Lattice \\
\hline 49.0 & Lattice & $49^{\mathrm{b}}$ & Lattice \\
\hline 54.6 & Lattice & $49^{\mathrm{b}}$ & Lattice \\
\hline 58.7 & Lattice & 59 & Lattice \\
\hline 71.0 & Lattice & 70 & Lattice \\
\hline
\end{tabular}

${ }^{\mathrm{a}}$ Data taken from Ref. 17.

${ }^{\mathrm{b}}$ No clear explanation was given by Rey-Lafon et al. as to why these modes were considered to be degenerate.

this trend is found to continue for other nitramine-based energetic materials, the role of degeneracies in phonon-phonon coupling could prove significant to the vibrational mechanisms of detonation initiation.

\section{Relevance to detonation}

Dlott and Fayer propose that the doorway modes, through which the energy of the shock-populated phonons can couple into the molecular degrees of freedom, are located in a frequency region approximately twice that of the highest energy optical phonon. Our data suggest that RDX is quite compatible with the model of Dlott and Fayer, where the $70.0 \mathrm{~cm}^{-1}$ optical phonon of RDX has its first overtone nearly degenerate with a $139.0 \mathrm{~cm}^{-1}$ molecular fundamental.

The unusual number of accidental degeneracies of the optical phonons has significant implications for the DlottFayer model of detonation initiation. Clearly, the degenerate energies of the optical phonons enhance their ability to couple. The accidentally degenerate optical phonons mediate all the Raman-active excitations of the low-energy molecular 
vibrations through phonon-phonon coupling. These lowenergy molecular modes are thought to be nitro-group deformation vibrations. ${ }^{17}$ The activation of these nitro-group deformations is of particular importance since it has been proposed that the initial bond broken in detonation is the $\mathrm{N}-\mathrm{N}$ bond that connects the nitro-group to the heterocyclic ring. ${ }^{10}$ However, these findings are also important to the Luty-Ordon-Eckhardt model where degeneracy plays an important role.

\section{CONCLUSIONS}

The single-crystal, polarized, Raman spectra for cyclotrimethylene trinitramine $(\mathrm{RDX})$ have been studied at ambient temperature and pressure over the region of 5-3400 $\mathrm{cm}^{-1}$. A consistent interpretation of the Raman spectra of RDX is provided by assignment of fundamental modes, overtones, and combination bands. Sixteen additional molecular vibrations were observed at ambient conditions. All 24 lattice optical phonons are also identified. A significant number of degenerate phonon energies of different modal symmetries are accidental. These accidentally degenerate optical phonons are relevant for coupling with low-lying, Raman-active molecular modes of RDX, an important requirement for the proposed vibrational mechanisms for detonation initiation. A similar analysis is planned for other solid energetic materials.

\section{ACKNOWLEDGMENTS}

Support of this research by the U.S. Office of Naval Research under Grant No. N000149810736 and by Pfizer, Inc. is gratefully acknowledged.

${ }^{1}$ Y. B. Zeldovich and Y. B. Raizer, Physics of Shock Waves and High Temperature Hydrodynamic Phenomena (Academic, New York, 1966); Ya. B. Zeldovich and A. S. Kompaneets, Theory of Detonation (Academic, New York, 1960).

${ }^{2}$ Y. B. Zeldovich, Zh. Eksp. Teor. Fiz. 10, 542 (1940).

${ }^{3}$ J. von Neumann, in Theory of Detonation Waves, John von Neumann Collected Works Vol. 6, edited by A. J. Taub (MacMillan, New York, 1942).

${ }^{4}$ W. Doring, Ann. Phys. 43, 417 (1943).
${ }^{5}$ D. D. Dlott and M. D. Fayer, J. Chem. Phys. 92, 3798 (1990).

${ }^{6}$ H. Kim and D. D. Dlott, J. Chem. Phys. 93, 1695 (1990).

${ }^{7}$ A. Tokmakoff, M. D. Fayer, and D. D. Dlott, J. Phys. Chem. 97, 1901 (1993).

${ }^{8}$ J. J. Gilman, Philos. Mag. B 71, 1057 (1995).

${ }^{9}$ T. Luty and C. J. Eckhardt, J. Am. Chem. Soc. 117, 2441 (1995).

${ }^{10}$ T. Luty, P. Ordon, and C. J. Eckhardt, J. Chem. Phys. 117, 1 (2002).

${ }^{11}$ S. D. McGrane and A. P. Shreve, J. Chem. Phys. 119, 5834 (2003).

${ }^{12}$ S. D. McGrane, J. Barber, and J. Quenneville, J. Phys. Chem. A 109, 9919 (2005).

${ }^{13}$ F. J. Owens and Z. Iqbal, J. Chem. Phys. 74, 4242 (1981).

${ }^{14}$ L. L. Stevens, J. J. Haycraft, and C. J. Eckhardt, Cryst. Growth Des. 5, 2060 (2005).

${ }^{15}$ For more information on recent advances see A. Blanco, N. Mina, M. E. Castro, J. Castillo-Chara, and S. P. Hernandez-Rivera, Proc. SPIE 5794, 1281 (2005); G. D. Pitt et al., IEE Proc.: Sci., Meas. Technol. 152, 241 (2005); M. L. Lewis, I. R. Lewis, and P. R. Griffiths, Vib. Spectrosc. 38, 11 (2005); 38, 17 (2005); F. T. Docherty, P. B. Monaghan, C. J. McHugh, D. Graham, W. E. Smith, and J. M. Cooper, IEEE Sens. J. 5, 632 (2005); L. M. Ballesteros, G. M. Herrera, M. E. Castro, J. Briano, N. Mina, and S. P. Hernandez-Rivera, Proc. SPIE 5794, 1254 (2005); J. C. Carter, S. M. Angel, M. Lawrence-Snyder, J. Scaffidi, R. E. Whipple, and J. G. Reynolds, Appl. Spectrosc. 59, 769 (2005); J. F. Bertone, K. L. Cordeiro, J. M. Sylvia, and K. M. Spencer, Proc. SPIE 5403, 387 (2004); M. L. Lewis, I. R. Lewis, and P. R. Griffiths, Appl. Spectrosc. 58, 420 (2004); S. K. Sharma, J. N. Porter, A. K. Misra, H. W. Hubble, and P. Menon, Proc. SPIE 5154, 1 (2003); J. M. Sylvia, K. M. Spencer, and J. A. Janni, Journal of Process Analytical Chemistry 6, 146 (2001).

${ }^{16}$ M. Rey-Lafon, C. Trinquecoste, R. Cavagnat, and M.-T. Forel, J. Chim. Phys. Phys.-Chim. Biol. 68, 1533 (1971).

${ }^{17}$ M. Rey-Lafon, C. Trinquecoste, R. Cavagnat, and M.-T. Forel, J. Chim. Phys. Phys.-Chim. Biol. 68, 1573 (1971).

${ }^{18}$ B. M. Rice and C. F. Chabalowski, J. Phys. Chem. A 101, 8720 (1997).

${ }^{19}$ C. S. Choi and E. Prince, Acta Crystallogr., Sect. B: Struct. Crystallogr. Cryst. Chem. 28, 2857 (1972). A more recent X-ray determination by M. Singh and S. Kaur [Curr. Sci. 78, 1184 (2000)] was found to be in substantial agreement with the previous neutron diffraction study by Choi and Prince. The structure by Singh and Kaur was solved in the nonstandard $P c a b$ setting and has a nearly identical calculated density to that of Choi and Prince.

${ }^{20}$ R. J. Karpowicz and T. B. Brill, J. Phys. Chem. 88, 348 (1984).

${ }^{21}$ T. C. Damen, S. P. S. Porto, and B. Tell, Phys. Rev. 142, 570 (1966).

${ }^{22}$ Spex 1400 Operating, Maintenance, and Instruction Manual (Spex Industries, Metuchen, NJ, 1969).

${ }^{23}$ J. L. McHale, Molecular Spectroscopy (Prentice-Hall, Upper Saddle River, NJ, 1999).

${ }^{24}$ J. J. Haycraft, L. L. Stevens, and C. J. Eckhardt, J. Chem. Phys. 124, 24712 (2006).

${ }^{25}$ Z. Iqbal, S. Bulusu, and J. R. Autera, J. Chem. Phys. 60, 221 (1974). 